

\title{
Zero-temperature Properties of a Trapped Bose-Condensed Gas: Beyond the Thomas-Fermi Approximation
}

\author{
Emil Lundh ${ }^{1,2}$, C. J. Pethick ${ }^{3,4}$ and H. Smith ${ }^{2}$ \\ ${ }^{1}$ Department of Theoretical Physics, UmeäUniversity, S-90187 Sweden \\ ${ }^{2}$ Ørsted Laboratory, H. C. Ørsted Institute, Universitetsparken 5, DK-2100 Copenhagen $\varnothing$, Denmark \\ ${ }^{3}$ Nordita, Blegdamsvej 17, DK-2100 Copenhagen $\emptyset$, Denmark, \\ ${ }^{4}$ Department of Physics, University of Mlinois at Urbana-Champaign, 1110 West Green Street, Urbana IL $61801-3080$
}

(December 4, 1996)

\begin{abstract}
We consider the leading corrections to the Thomas-Fermi approach to the description of the properties of a magnetically trapped, Bose-condensed cloud of atoms. Simple analytical expressions are derived for the kinetic energy in terms of an effective cut-off length, which we calculate numerically by considering the one-dimensional problem for a linear-ramp potential. We also determine the lowest angular velocity at which it is energetically favorable for a vortex to enter the cloud. For large clouds, our results are in excellent agreement with available numerical calculations.
\end{abstract}

PACS numbers: 03.75.Fi,03.65.Db,05.30.Jp,32.80.Pj

\section{INTRODUCTION}

The realization by Anderson et al. [1] of Bose condensation in magnetically trapped ${ }^{87} \mathrm{Rb}$ gas has generated considerable excitement and stimulated interest in the ground-state properties of the dilute Bose gas. For a system so dilute that depletion of the condensate can be neglected, the wave function, $\psi(\vec{r})$, of the condensed state in an external potential $V(\vec{r})$ is given by the Gross-Pitaevskii (GP) equation [2]

$$
\left[-\frac{\hbar^{2}}{2 m} \nabla^{2}+V(\vec{r})+\frac{4 \pi \hbar^{2} a}{m}|\psi(\vec{r})|^{2}\right] \psi(\vec{r})=\mu \psi(\vec{r}),
$$

where $\mu$ is the chemical potential. The effective two-body interaction is $4 \pi \hbar^{2} a / m$, where $a$ is the scattering length and $m$ is the particle mass. It was shown recently by Baym and Pethick [3] that the ground-state properties of the trapped Bose gas with repulsive interactions may be described quite accurately for a sufficiently large number of particles by a Thomas-Fermi (TF) approach, in which the kinetic energy is neglected. The density is then given by

$$
|\psi(\vec{r})|^{2}=\frac{m}{4 \pi \hbar^{2} a}(\mu-V(\vec{r})) \text { for } V(\vec{r}) \leq \mu .
$$

For a non-rotating cloud this approach does not, however, take properly into account the decay of the wave function near the outer edge of the cloud, and the Thomas-Fermi wave function consequently leads to unphysical behavior for some properties, most notably the kinetic energy. This is easily seen since at the outer edge the wave function varies as the square root of the distance from the turning point, and therefore its derivative varies inversely as the square root of the distance. As a consequence, the kinetic energy diverges logarithmically as the end point of the integration approaches the turning point.

In this paper we show how to evaluate the leading corrections to the Thomas-Fermi approach for large drops. We shall consider two different cases. The first is that of non-rotating drops, where the kinetic energy is all-important for the form of the wave function at the outer edge of a cloud of atoms. The second situation where the role of the kinetic energy is crucial is in determining the structure of a vortex line in a cloud of atoms. In both cases the basic philosophy will be the same: the Thomas-Fermi approximation is good except in a limited volume of space where the kinetic energy operator is important. In the first case this region is close to the classical turning points at the edge of the cloud, while in the latter case it is the region near the vortex line. Our approach is to graft solutions of the GP equation onto those of the TF equation, thereby enabling us to derive analytical expressions for quantities of interest. These will be compared with the results of numerical calculations for both these situations that have been performed by Dalfovo and Stringari [4].

The plan of the paper is as follows: In Sec.II we consider non-rotating clouds, and show that the structure of the wave function at the outer edge is given by the solution of the GP equation for motion in a linear potential. We then apply this result to calculate the kinetic energy of clouds of atoms in an anisotropic harmonic oscillator potential. In Sec.III we discuss properties of vortex lines, and derive expressions for the critical angular velocity at which it becomes energetically favorable for a vortex line to enter a cloud. 


\section{NON-ROTATING CLOUDS}

The basic idea of our approach is that for large drops, the wave function is close to the Thomas-Fermi result, except near classical turning points, where the kinetic energy term cannot be neglected. As one can see from the differential equation, the characteristic length associated with the structure near the turning point is of order $\left(\hbar^{2} / 2 m F\right)^{1 / 3}$, where $F=|\vec{\nabla} V|$ is the force acting on a particle at the turning point. This length is the same as that which enters the Airy function solution for the wave function of a particle in a linear-ramp potential. Thus if the dependence of the wave function is small in directions perpendicular to $\vec{\nabla} V$, the GP equation in the vicinity of the classical turning point reduces to that for a linear-ramp potential [5]. We therefore solve the non-linear GP equation for a linear-ramp potential. When the kinetic energy is included, the wave function acquires a tail and extends smoothly beyond the classical turning point. We shall consider the effect of this tail on the momentum distribution.

\section{A. The linear-ramp potential}

For a linear-ramp potential the GP equation is

$$
\left[-\frac{\hbar^{2}}{2 m} \frac{d^{2}}{d x^{2}}+F x+\frac{4 \pi \hbar^{2} a}{m}|\psi(x)|^{2}\right] \psi(x)=\mu \psi(x)
$$

where the coordinate $x$ measures distances in the direction of $-\vec{\nabla} V$, and the origin is chosen to be at the classical turning point.

After introducing a scaled length variable $y=x / \delta$ where $\delta$ is given by $\hbar^{2} / 2 m \delta^{2}=F \delta$, or $\delta=\left(\hbar^{2} / 2 m F\right)^{1 / 3}$ and a scaled wave function given by $\Psi=\psi / b$ where $b^{2}=F m \delta / 4 \pi \hbar^{2} a$ or $b^{2}=\left(2 F m / \hbar^{2}\right)^{2 / 3} / 8 \pi a$, we obtain

$$
\Psi^{\prime \prime}=y \Psi+\Psi^{3},
$$

where the prime denotes differentiation with respect to $y$. The corresponding Thomas-Fermi approximation is

$$
\Psi=\sqrt{-y} \text { for } y \leq 0, \quad \Psi=0 \text { for } y>0 .
$$

Note that the derivative of the TF wave function diverges as $y \rightarrow 0^{-}$, as in the general case.

Before we discuss the mumerical solution of $(3)$ we consider the behavior for $|y| \gg 1$.

a) $y \gg 1$.

Here we may linearize (3) and obtain by neglecting the cubic term the asymptotic solution

$$
\Psi \simeq \frac{C}{y^{1 / 4}} e^{-2 y^{3 / 2} / 3}
$$

which is just the asymptotic behavior of the Airy function.

b) $y \ll-1$

Here the $\mathrm{TF}$ solution $\Psi \simeq \sqrt{-y}$ is approximately valid. In order to determine the leading correction to this, we write $\Psi=\Psi_{0}+\Psi_{1}$ and linearize (3), thereby finding

$$
-\Psi_{1}^{\prime \prime}+y \Psi_{1}+3 \Psi_{0}^{2} \Psi_{1}=\Psi_{0}^{\prime \prime}
$$

Using $\Psi_{0}^{2}=-y$ and $\Psi_{0}^{\prime \prime}=1 / 4 y \sqrt{-y}$ we arrive at

$$
\Psi_{1} \simeq-\frac{1}{8 y^{2} \sqrt{-y}}
$$

where the second derivative of $\Psi_{1}$ has been neglected, since it contributes to terms of higher order in $1 / y$. The asymptotic solution is thus

$$
\Psi=\sqrt{-y}\left(1+\frac{1}{8 y^{3}}\right)
$$

The numerical solution is shown in Fig.1, where we also show the asymptotic behavior for large $|y|$. From the numerical result we find that the constant $C$ entering (5) is approximately equal to 0.3971 . 
Let us now calculate the kinetic energy associated with this solution. One question that immediately comes up is which operator one should use to do this. Two possibilities are $\left(\hbar^{2} / 2 m\right) \int d^{3} r|\vec{\nabla} \psi|^{2}$ and $-\left(\hbar^{2} / 2 m\right) \int d^{3} r \psi^{*} \nabla^{2} \psi$, and provided the wave function vanishes or has zero gradient on the boundary of the volume over which the integration is performed, the two expressions will lead to identical results. However, for the ramp potential the product of the wave function and its gradient tends to a constant for large negative values of $y$. This apparent difficulty is removed by recognizing that, in realistic physical situations, the potential will not be a linear ramp in the whole of space, and therefore one has to match the solution for the linear ramp to the wave function for some other more general potential, for example that for a harmonic oscillator in the problem of experimental interest. The answer for the total kinetic energy will not depend on the form of the operator used, provided that $\psi^{*} \nabla \psi$ vanishes rapidly enough at large distances, but how one assigns the kinetic energy to various parts of space will depend on the choice of operator. This reflects the fact that the kinetic energy density operator is not uniquely defined.

We now evaluate the contribution to the kinetic energy for the wave function for the linear ramp, and for definiteness we shall write this as

$$
\frac{<p^{2}>}{2 m}=-\frac{\hbar^{2}}{2 m} \int d^{3} r \psi^{*} \nabla^{2} \psi
$$

Let us first use the Thomas-Fermi wave function (4) for the calculation of the kinetic energy. We expect this to be valid in the region $x \ll-\delta$. Because of the square-root behavior of $(4)$ the integration must be cut off at a distance $l$ (of order $\delta$ ) from the turning point. In evaluating the mean value of the square of the momentum, $p^{2}$, using (4), we introduce a lower cut-off at $x=-L$ and integrate from $-L$ to $x=-l$,

$$
<p^{2}>=-\hbar^{2} \frac{\int_{-L}^{-1} d x \psi \psi^{\prime \prime}}{\int_{-L}^{-l} d x \psi^{2}} \simeq \frac{\hbar^{2}}{2 L^{2}} \ln \frac{L}{\delta}+\mathcal{O}\left(\frac{\hbar^{2}}{L^{2}}\right)
$$

The term of order $\hbar^{2} / L^{2}$ may be evaluated from the numerical solution to (3) which we show in Fig.1. The mean value of the square of the momentum is obtained from

$$
<p^{2}>=-\hbar^{2} \frac{\int_{-L}^{\infty} d x \psi \psi^{\prime \prime}}{\int_{-L}^{\infty} d x \psi^{2}}
$$

In Fig. 2 we plot the dependence of $\left\langle p^{2}>L^{2} / \hbar^{2}\right.$ on $\ln (L / \delta)$. For large values of $\ln (L / \delta)$ the dependence is fitted by the linear function $\frac{1}{2} \ln (L / \delta)-0.2872=\frac{1}{2} \ln (L / 1.776 \delta)$.

We conclude that one obtains the correct asymptotic behavior of the kinetic energy, if one cuts off the integration in the Thomas-Fermi approach at

$$
l=1.776 \delta
$$

where we have restored the length scale introduced above (3). As we shall demonstrate below, the same effective cut-off may be used for calculating the kinetic energy in more general situations.

Note that when $L$ is slightly less than $\delta$ the contribution to $<p^{2}>$ exhibited in Fig.2 turns negative, the wave function and its second derivative having the same sign over most of the region of integration. This is not a problem, since the totalkinetic energy for a physically acceptable wave function will be positive. Here, however, we are interested only in the region of large in $L$ in order to determine the effective cut-off length $l$.

\section{B. The isotropic harmonic oscillator}

We now turn to the system of physical interest, a cloud of atoms which is trapped in a three-dimensional harmonic oscillator potential. For simplicity, we first consider the isotropic case, where the potential is $V(r)=m \omega^{2} r^{2} / 2$, leaving the anisotropic case to Sect.IIC. The GP equation for the ground state wave function is

$$
\left[-\frac{\hbar^{2}}{2 m r^{2}} \frac{d}{d r}\left(r^{2} \frac{d}{d r}\right)+\frac{1}{2} m \omega^{2} r^{2}+\frac{4 \pi \hbar^{2} a}{m}|\psi(r)|^{2}\right] \psi(r)=\mu \psi(r)
$$

By the substitution $\chi=r \psi$ we obtain

$$
-\frac{\hbar^{2}}{2 m} \frac{d^{2} \chi}{d r^{2}}+\frac{1}{2} m \omega^{2}\left(r^{2}-R^{2}\right) \chi(r)+\frac{4 \pi \hbar^{2} a}{m r^{2}}|\chi(r)|^{2} \chi(r)=0,
$$


where $\mu=m \omega^{2} R^{2} / 2$. The TF solution is

$$
\chi_{\mathrm{TF}}=\frac{m \omega r \sqrt{R^{2}-r^{2}}}{\sqrt{8 \pi a \hbar^{2}}} .
$$

By approximating $r^{2}-R^{2} \simeq 2 R(r-R)$ and replacing $r$ by $R$ in the interaction term we arrive at an equation of the form (3). The characteristic length scale $\delta$ is obtained by equating the energy $\hbar^{2} / 2 m \delta^{2}$ to the force $m \omega^{2} R$ times the distance $\delta$, resulting in $\delta^{3}=\hbar^{2} / 2 m^{2} \omega^{2} R$ or

$$
\frac{\delta}{R}=\frac{1}{2}\left(\frac{\hbar \omega}{\mu}\right)^{2 / 3}
$$

where we have used the fact that $\mu=m \omega^{2} R^{2} / 2$.

As before we may work out the correction to the TF wave function for $r$ much less than $R$. Writing $\chi=\chi_{0}+\chi_{1}$ we obtain to leading order in $r / R$ that the corresponding value of $\psi(=\chi / r)$ is reduced by the factor

$$
1-\frac{3 \hbar^{2}}{2 m^{2} \omega^{2} R^{4}}
$$

at the origin, relative to the value of the TF wave function for the same value of $\mu$.

For the TF wave function the mean square momentum is

$$
<p^{2}>=-\hbar^{2} \frac{\int_{0}^{R-1} d r \chi \chi^{\prime \prime}}{\int_{0}^{R} d r \chi^{2}} \simeq \frac{\hbar^{2}}{R^{2}}\left(\frac{15}{4} \ln \frac{2 R}{l}-\frac{5}{2}\right)
$$

where we have introduced a cut-off at $R-l$ as in one dimension. In Fig.3 we exhibit the result of numerically integrating (14) and calculating $\left\langle p^{2}\right\rangle$ by integrating from zero to infinity. These numerical results are compared with the simple Thomas-Fermi formula (17) with $l$ given by the result (12) obtained from the linear ramp in one dimension, while the characteristic length $\delta$ is given by (16). It is seen that the simple formula agrees well with the numerical result for $\ln (R / \delta)$ greater than 3 , the relative difference being less than $2.5 \%$. For the ${ }^{87} \mathrm{Rb}$ experiment this corresponds to the number of atoms exceeding $3 \cdot 10^{5}$. Using a somewhat different approach Dalfovo et al. [5] have independently obtained a result similar to (17).

\section{The anisotropic harmonic oscillator}

In the experiments reported in [1] the cloud of ${ }^{87} \mathrm{Rb}$-atoms was magnetically confined in a TOP trap, an effective three-dimensional harmonic oscillator potential with angular frequencies $\omega_{z}^{0}$ in the axial direction (taken to be the $z$-axis) and $\omega_{\perp}^{0}=\omega_{z}^{0} / \sqrt{8}$ in the transverse direction (the $x-y$ plane). The corresponding characteristic lengths are denoted by $a_{z}=\left(\hbar / m \omega_{z}\right)^{1 / 2}$ and $a_{\perp}=\left(\hbar / m \omega_{\perp}\right)^{1 / 2}, m$ being the mass of a ${ }^{87} \mathrm{Rb}$-atom.

In the following we perform an analytical calculation of the mean kinetic energy for such a potential, in terms of the effective cut-off length $l$ derived in Sect.IIA. The kinetic energy is determined as a function of the parameter $\lambda$ specifying the ratio between the axial and the transverse oscillator frequency according to

$$
\omega_{z}=\lambda \omega_{\perp}
$$

Finally, we evaluate our expression for the kinetic energy for the case $\lambda=\sqrt{8}$ appropriate to [1] and compare our results with the numerical calculations performed in [4].

In cylindrical coordinates the potential $V(\rho, z)$ is thus given by

$$
V=\frac{1}{2} m \omega_{\perp}^{2}\left(\rho^{2}+\lambda^{2} z^{2}\right)
$$

The corresponding Thomas-Fermi wave function is

$$
\psi=A \sqrt{R^{2}-\rho^{2}-\lambda^{2} z^{2}}
$$

with $A=\left(8 \pi a_{\perp}^{4} a\right)^{1 / 2}$, while $R$ is related to the chemical potential $\mu$ by $\mu=m R^{2} \omega_{\perp}^{2} / 2$. Using the wave function (20) we obtain 


$$
-\psi \nabla^{2} \psi=A^{2} \frac{\left(2+\lambda^{2}\right) R^{2}-\left(1+\lambda^{2}\right) \rho^{2}-2 \lambda^{2} z^{2}}{R^{2}-\rho^{2}-\lambda^{2} z^{2}}
$$

In evaluating the total kinetic energy we first integrate (21) over $\rho$ from 0 to $\rho_{0}(z)-l(z)$, with $\rho_{0}(z)=\sqrt{R^{2}-\lambda^{2} z^{2}}$, while $l(z)$ is the $z$-dependent effective cut-off length. The $z$-dependence of $l(z)$ arises from two sources: First, in the direction perpendicular to the surface the cut-off length is inversely proportional to the cube root of the magnitude of the potential gradient, $|\nabla V(\vec{r})|$, evaluated at the surface $\rho=\rho_{0}(z)$ and hence to $\left(1+\left(\lambda^{4}-\lambda^{2}\right)(z / R)^{2}\right)^{-1 / 6}$. Secondly, since we integrate over $\rho$, the effective cut-off is increased by the inverse of the cosine of the angle between the $\rho$-axis and the direction of the potential gradient at the surface. Together, these two effects imply that

$$
l(z)=l(0) \frac{\left(1-\left(\lambda^{2}-1\right) \lambda^{2}(z / R)^{2}\right)^{1 / 3}}{\left(1-\lambda^{2}(z / R)^{2}\right)^{1 / 2}}
$$

In calculating the kinetic energy we first integrate over $\rho$. The ensuing $z$-integration of $\ln l(z)$ involves elementary integrals. The final result for the mean kinetic energy per particle may be written as

$$
<\frac{p^{2}}{2 m}>=\frac{\hbar^{2}}{2 m R^{2}} F\left(\lambda, N, a, a_{\perp}\right)
$$

where

$$
F=\frac{5}{4}\left(\lambda^{2}+2\right) \ln \frac{2 R}{l(0) \lambda^{2 / 3}}-\frac{5}{18}\left(6 \frac{\tan ^{-1} \sqrt{\lambda^{2}-1}}{\sqrt{\lambda^{2}-1}}+2 \lambda^{2}+1\right),, \lambda>1,
$$

for oblate traps and

$$
F=\frac{5}{4}\left(\lambda^{2}+2\right) \ln \frac{2 R}{l(0) \lambda^{2 / 3}}-\frac{5}{18}\left(\frac{3}{\sqrt{1-\lambda^{2}}} \ln \frac{1+\sqrt{1-\lambda^{2}}}{1-\sqrt{1-\lambda^{2}}}+2 \lambda^{2}+1\right),, \lambda<1,
$$

for prolate traps. For an isotropic trap these results reduce to the one given in the previous sub-section. The integrations leading to the results (23-25) can alternatively be performed by scaling the coordinates associated with the principal axes of the ellipsoid so that the region of integration becomes the unit sphere.

In evaluating (23-25) it is convenient to relate $R$ and $\delta$ to the number of atoms $N$, the scattering length $a$ and the oscillator parameters $a_{\perp}$ and $\lambda$ according to

$$
\frac{R}{a_{\perp}}=\left(\frac{15 N \lambda a}{a_{\perp}}\right)^{1 / 5}
$$

and

$$
\frac{R}{\delta}=2^{1 / 3}\left(\frac{15 N \lambda a}{a_{\perp}}\right)^{4 / 15} .
$$

In Fig.(4) we show our result for the kinetic energy as a function of $N$ for the parameter values $\lambda=\sqrt{8}$ and $a / a_{\perp}=4.33 \cdot 10^{-3}$ appropriate for the experiment of [1], together with the results of the numerical calculations of [4]. For the case of $N=10^{4}$, our approximate expression for the kinetic energy per particle gives $0.38 \hbar \omega_{\perp}$ which compares favorably with the result of the numerical calculations, $0.45 \hbar w_{\perp}$.

\section{THE ENERGY OF A VORTEX}

We now calculate the energy of a single vortex in a Bose-condensed cloud. This quantity is important for estimating the lowest angular velocity for which it is favorable for a vortex to enter the cloud. In a uniform medium, the energy per unit length of a vortex with a single quantum of circulation $2 \pi \hbar / \mathrm{m}$ lying along the axis of a cylindrical container of radius $b$ is given by

$$
\epsilon=\pi n \frac{\hbar^{2}}{m} \ln \frac{1.464 b}{\xi}
$$


where $\xi$ is the coherence length. This result was first obtained by Ginzburg and Pitaevskii [6]. The coherence length $\xi$ is defined in the usual manner by

$$
\frac{\hbar^{2}}{2 m \xi^{2}}=\frac{4 \pi a \hbar^{2} n}{m}
$$

where $n=|\psi|^{2}$ is the density of the uniform medium. For a large cloud of atoms of radius $R$ this implies that the coherence length at the center is given by

$$
\frac{\xi}{R}=\frac{\hbar \omega}{2 \mu}
$$

since the chemical potential is related to the central density $n(0)$ by $\mu=4 \pi \hbar^{2}$ an $(0) / m$.

If the characteristic dimensions of the cloud are large compared with the coherence length at the center of the cloud, we may determine the energy in a simple way by using the result (28) to calculate the energy out to a radius $\rho_{1}$ satisfying $\xi \ll \rho_{1} \ll R$ and then calculate the energy at larger distances in a purely hydrodynamic manner. First of all, let us consider the two-dimensional problem, in which we neglect the $z$-dependence. The energy per unit length is then given by

$$
\epsilon=\pi n_{0} \frac{\hbar^{2}}{m} \ln \frac{1.464 \rho_{1}}{\xi_{0}}+\frac{1}{2} \int_{\rho_{1}}^{R} m n(\rho) v^{2}(\rho) 2 \pi \rho d \rho .
$$

Here $n_{0}$ is the particle density for $\rho \rightarrow 0$ in the absence of a vortex, while $\xi_{0}$ is the coherence length evaluated for that density. Since the velocity $v$ is given in magnitude by $\hbar / m \rho$ and the density in a harmonic trap varies as $\left(1-\rho^{2} / R^{2}\right)$ in the Thomas-Fermi approximation, one finds

$$
\epsilon=\pi n_{0} \frac{\hbar^{2}}{m} \ln \frac{1.464 \rho_{1}}{\xi_{0}}+\pi n_{0} \frac{\hbar^{2}}{m} \int_{\rho_{1}}^{R} \frac{r d r}{r^{2}}\left(1-\frac{r^{2}}{R^{2}}\right) \simeq \pi n_{0} \frac{\hbar^{2}}{m}\left(\ln \frac{1.464 R}{\xi_{0}}-\frac{1}{2}\right),
$$

where the integral has been evaluated for $\rho_{1} \ll R$, with terms of higher order in $\rho_{1} / R$ being neglected. The logarithmic term is the result for a medium of uniform density, while the $-1 / 2$ reflects the lowering of the kinetic energy due to the reduction of particle density caused by the presence of the trapping potential. Thus the energy per unit length is given by an expression similar to $(28)$ but with a different numerical constant $1.464 / e^{1 / 2}=0.888$,

$$
\epsilon=\pi n_{0} \frac{\hbar^{2}}{m} \ln \frac{0.888 R}{\xi_{0}}
$$

The angular momentum $\mathcal{L}$ per unit length is just $\hbar$ times the total number of particles per unit length. For $R \gg \xi$ the latter may be evaluated in the Thomas-Fermi approximation, and one finds

$$
\mathcal{L}=n_{0} \hbar \int_{0}^{R}\left(1-\frac{\rho^{2}}{R^{2}}\right) 2 \pi \rho d \rho=\frac{1}{2} n_{0} \pi R^{2} \hbar
$$

The critical angular velocity $\Omega_{c 1}$ for a vortex to be energetically favorable is given by the condition $\epsilon-\Omega_{c 1} \mathcal{L}=0$, and therefore, by combining (33) and (34),

$$
\Omega_{c 1}=2 \frac{\hbar}{m R^{2}} \ln \frac{0.888 R}{\xi_{0}} .
$$

Let us now consider the three-dimensional problem. If the semi-axis, $Z$, of the cloud in the $z$-direction is much greater than the coherence length, one may estimate the energy of the cloud by adding the energy of horizontal slices of the cloud. The total energy is then given by (33), integrated over the vertical extent of the cloud,

$$
E=\frac{\pi \hbar^{2}}{m} \int_{-Z}^{Z} d z n_{0}(z) \ln \frac{0.888 R(z)}{\xi(z)} .
$$

For a harmonic trap $n_{0}(z)=n_{00}\left(1-z^{2} / Z^{2}\right)$, while $R(z)=R\left(1-z^{2} / Z^{2}\right)^{1 / 2}$ and $\xi(z)=\xi(z=0)\left(n_{00} / n_{0}(z)\right)^{1 / 2}$. The energy is then given simply as 


$$
E=\frac{\pi \hbar^{2} n_{00}}{m} \int_{-Z}^{Z} d z\left(1-\frac{z^{2}}{Z^{2}}\right) \ln \left(\frac{0.888 R}{\xi_{0}}\left(1-\frac{z^{2}}{Z^{2}}\right)\right)
$$

Using the fact that $\int_{0}^{1} d y\left(1-y^{2}\right) \ln \left(1-y^{2}\right)=(12 \ln 2-10) / 9$ we obtain the final result

$$
E=\frac{4 \pi n_{00}}{3} \frac{\hbar^{2}}{m} Z \ln \frac{0.671 R}{\xi_{0}}
$$

The total angular momentum is

$$
\mathcal{L}=n_{00} \hbar \int\left(1-\frac{\rho^{2}}{R^{2}}-\frac{z^{2}}{Z^{2}}\right) d z 2 \pi \rho d \rho=\frac{8 \pi}{15} n_{00} R^{2} Z \hbar
$$

and therefore the lower critical angular velocity is given by

$$
\Omega_{c 1}=\frac{5}{2} \frac{\hbar}{m R^{2}} \ln \frac{0.671 R}{\xi_{0}} .
$$

When this expression for the critical angular velocity is evaluated In Fig.(5) we show the results for the critical angular velocity for the parameters $\lambda=\sqrt{8}$ and $a / a_{\perp}=4.33 \cdot 10^{-3}$ appropriate for the experiment of Ref. [1], together with the results of the numerical integrations of the GP equation [4]. For $N=10^{4}$ we find that the critical angular velocity is $0.323 \omega_{\perp}$, which agrees extremely well with the result of the numerical calculations.

\section{DISCUSSION}

In this paper we have derived analytical expressions for the kinetic energy and lower critical angular velocity for large clouds of Bose-condensed atoms. For experimentally realizable conditions, our results are a good approximation to those obtained by solving the Gross-Pitaevskii equation numerically. Our methods for calculating the kinetic energy may readily be applied to more general traps, such as harmonic traps with no axis of symmetry.

We remark that our starting point, the Gross-Pitaevskii equation, may be used with confidence since, for the conditions in experiments to date, depletion of the condensate due to particle interactions is extremely small. This may easily be seen from the fact that for the uniform Bose gas, the fractional depletion of the zero momentum state is $\left(8 /\left(3 \pi^{1 / 2}\right)\right)\left(n_{0} a^{3}\right)^{1 / 2}$, which is of the order of one per cent.

For the conditions in the experiment [1] the agreement between the analytical results and the results of numerical integrations is even better for the critical angular velocity than it is for the kinetic energy. This is a consequence of the fact that the largest contribution to the kinetic energy comes from motion in which the cloud is thinnest, the $z$-direction, for which our semiclassical wave function is less accurate than for the motion in the other directions which dominates the result for the critical angular velocity.

\section{ACKNOWLEDGEMENT}

We are grateful to F. Dalfovo and S. Stringari for providing us with numerical values of their results for the critical rotational velocity.

[1] M.H. Anderson, J.R. Ensher, M.R. Matthews, C.E. Wieman, and E.A. Cornell, Science 269, 198 (1995).

[2] L.P. Pitaevskii, Zh. Eksp. Teor. Fiz. 40, 646 (1961) [Sov. Phys.-JETP 13, 451 (1961)]; E.P. Gross, Nuovo Cimento 20, 454 (1961), J. Math. Phys. 4, 195 (1963).

[3] G. Baym and C.J. Pethick, Phys. Rev. Lett. 76,6 (1996).

[4] F. Dalfovo and S. Stringari, Phys. Rev. A 53, 2477 (1996)

[5] Independently, F. Dalfovo, L.P. Pitaevskii and S. Stringari, Phys. Rev. A 54, 4213 (1996), have presented similar arguments, and have calculated the kinetic energy of a cloud in an isotropic harmonic trap.

[6] V.L. Ginzburg and L.P. Pitaevskii, Zh. Eksp. Teor. Fiz. 34, 1240 (1958) [Sov. Phys.-JETP 7, 858 (1958)]. 
Figure captions

Fig. 1. The numerical solution for the linear-ramp potential (full line) together with the various asymptotic solutions.

Fig. 2. The figure shows the dependence of $\left\langle p^{2}>L^{2} / \hbar^{2}\right.$ on $\ln (L / \delta)$. For large values of $\ln (L / \delta)$ the dependence is linear and given approximately by $\frac{1}{2} \ln (L / 1.776 \delta)$.

Fig. 3. A comparison of analytical and numerical results for the kinetic energy of the isotropic three-dimensional oscillator.

Fig. 4. The kinetic energy per particle, Eq. (23), in units of $\hbar \omega_{\perp}$ as a function of the particle number $N$ for parameters appropriate to the ${ }^{87} \mathrm{Rb}$ experiment: $\lambda=\sqrt{8}$ and $a / a_{\perp}=4.33 \cdot 10^{-3}$. The data points are the results of the numerical integrations of Ref. [4].

Fig. 5. The critical angular velocity, Eq. (40), in units of $\omega_{\perp}$ as a function of the particle minitrer .1 for parameters appropriate to the ${ }^{87} \mathrm{Rb}$ experiment: $\lambda=\sqrt{8}$ and $a / a_{\perp}=4.33 \cdot 10^{-3}$. Again, the data muts are taken from the numerical calculations of Ref. [4]. 
Fig.1

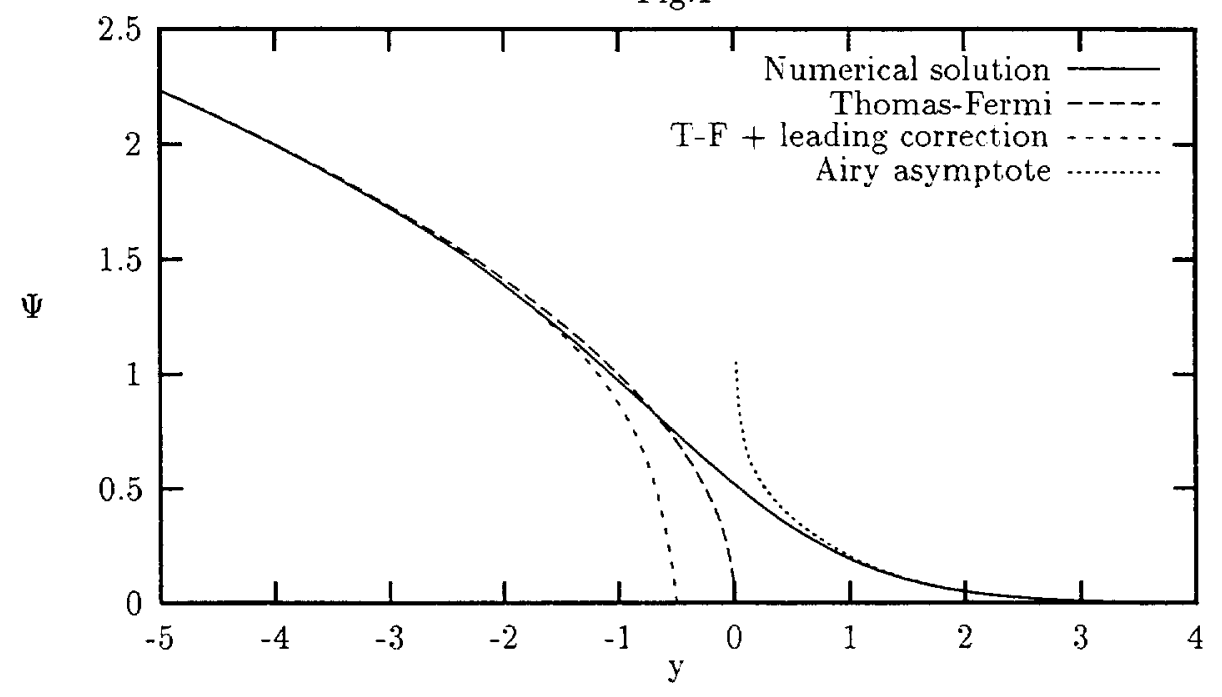


Fig. 2

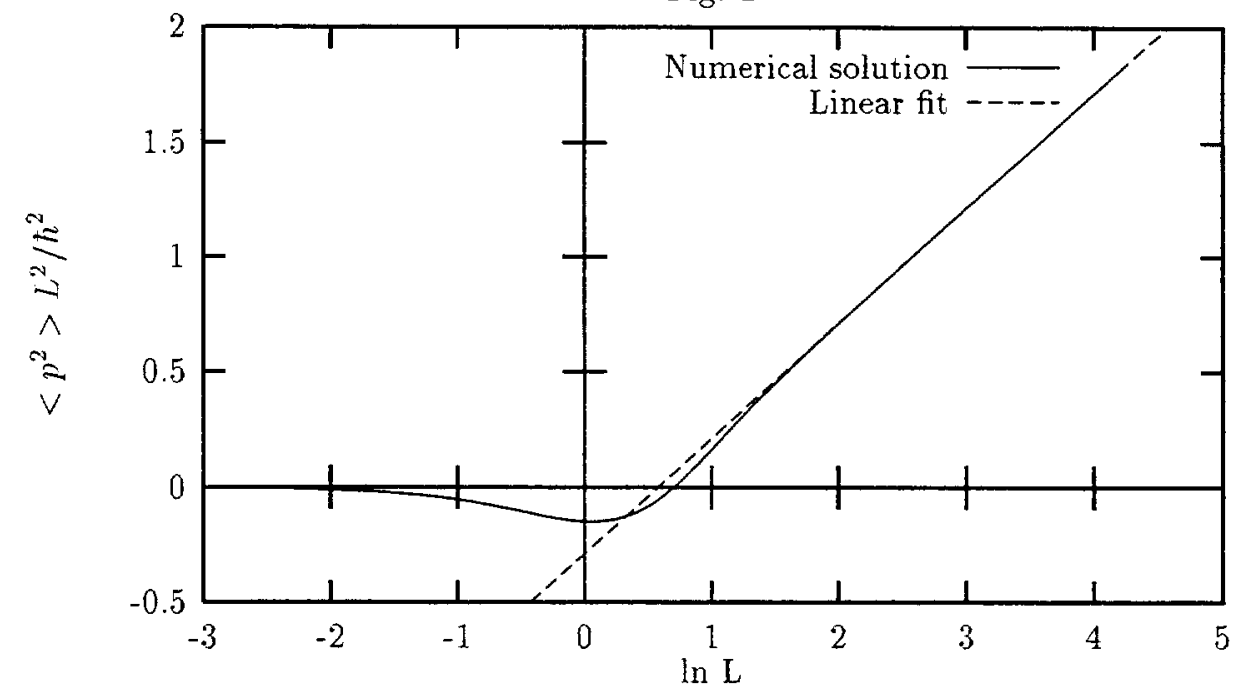


Fig.3

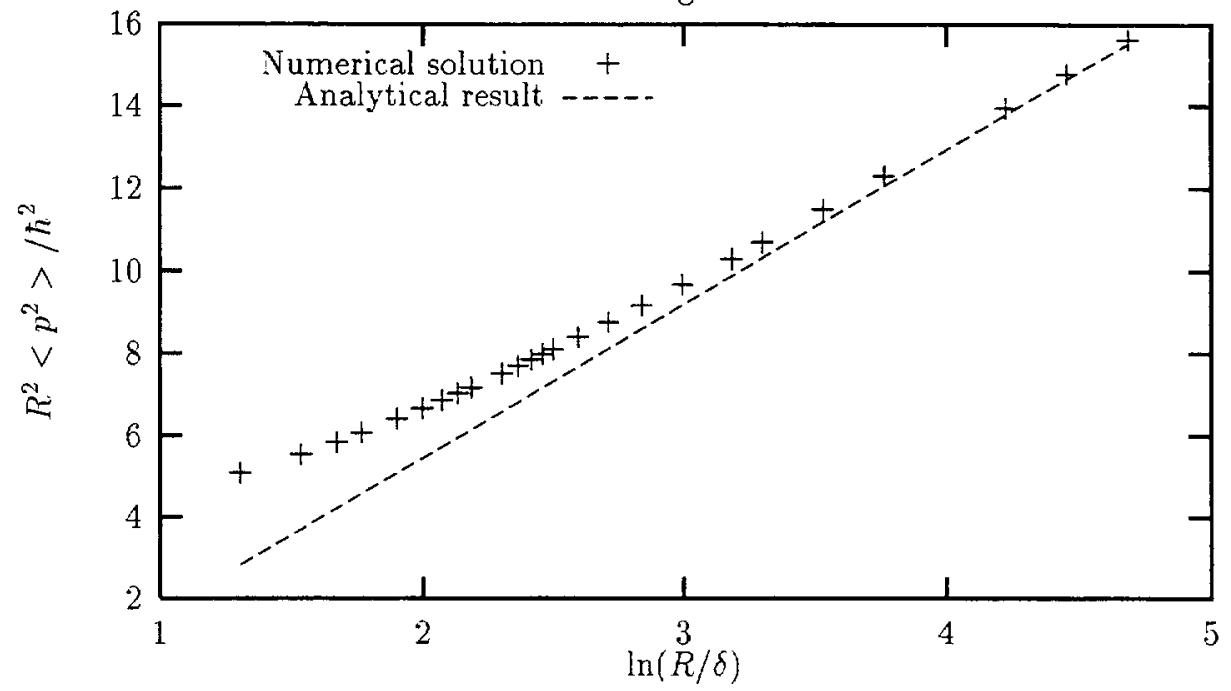


fig. 4

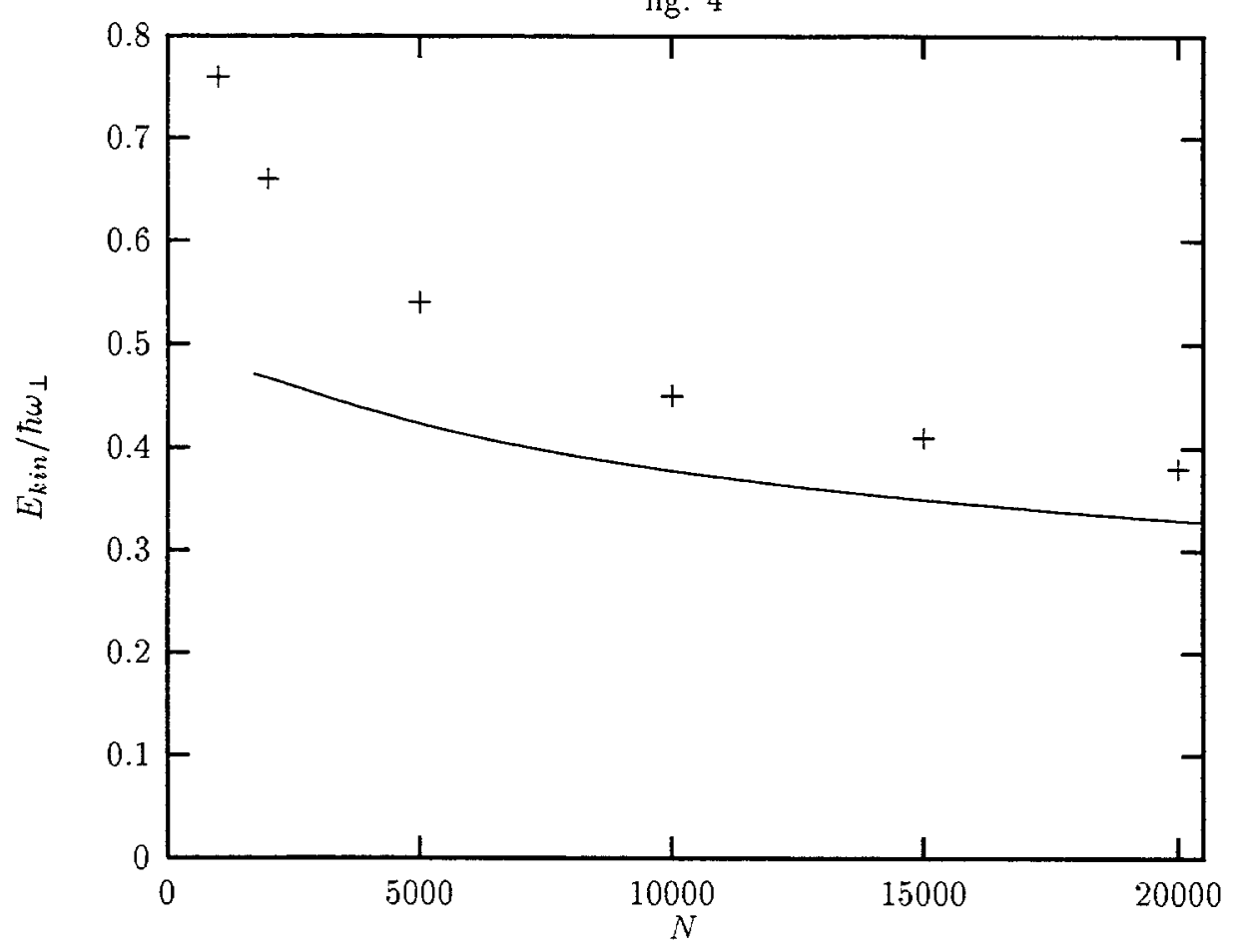


fig. 5

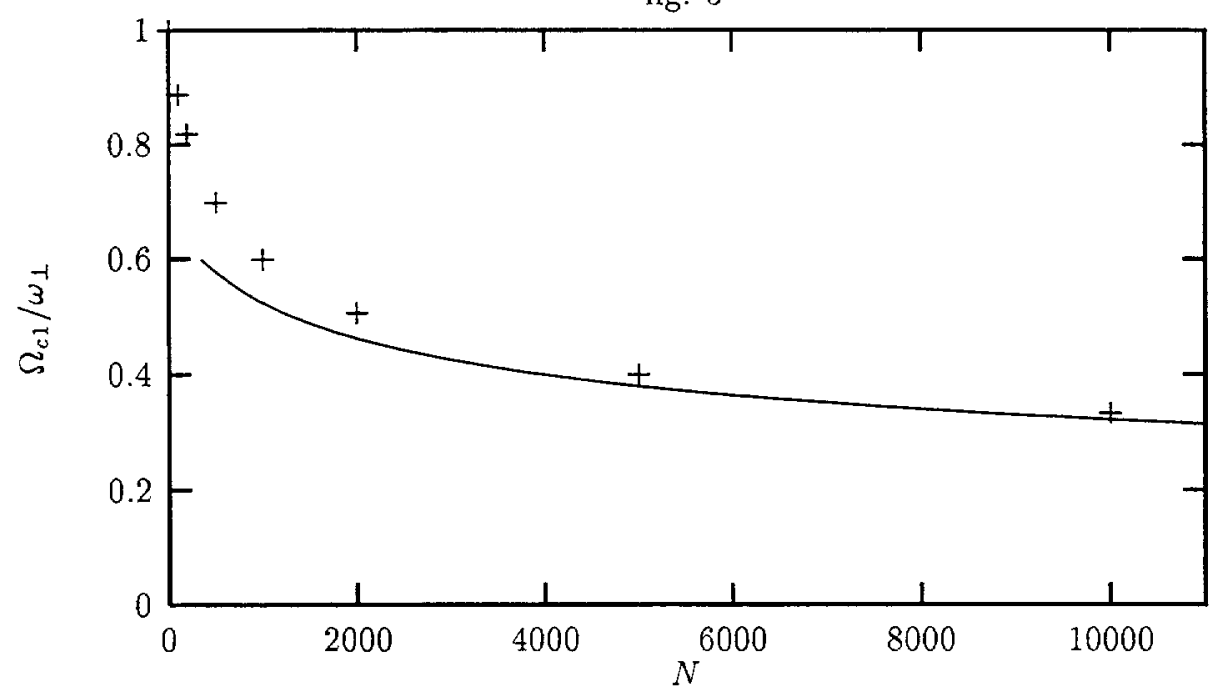


